\title{
Last technology and results from the IOTA interferometer
}

Ettore Pedretti, Wesley A. Traub, John D. Monnier, Peter A. Schuller, Sam Ragland, et al.

Ettore Pedretti, Wesley A. Traub, John D. Monnier, Peter A. Schuller, Sam Ragland, Jean-Philippe Berger, Rafael Millan-Gabet, Gary Wallace, Michael Burke, Marc G. Lacasse, Nathalie D. Thureau, Nathaniel Carleton, "Last technology and results from the IOTA interferometer," Proc. SPIE 7013, Optical and Infrared Interferometry, 70132V (28 July 2008); doi: $10.1117 / 12.789751$

SPIE Event: SPIE Astronomical Telescopes + Instrumentation, 2008, Marseille, France 


\title{
Last technology and results from the IOTA interferometer
}

\author{
Ettore Pedretti ${ }^{a \dagger}$, Wesley A. Traub ${ }^{b}$, John D. Monnier ${ }^{c}$, Peter A. Schuller ${ }^{d}$, Sam Ragland ${ }^{e}$, \\ Jean-Philippe Berger ${ }^{f}$, Rafael Millan-Gabet ${ }^{g}$, Gary Wallace ${ }^{h}$, Michael Burke ${ }^{i}$, Marc G. \\ Lacasse $^{i}$, Nathalie D. Thureau ${ }^{a}$, and Nathaniel Carleton ${ }^{i}$ \\ ${ }^{a}$ University of St Andrews, School of Physics and Astronomy, St Andrews, Fife, KY16 0UZ, \\ Scotland \\ ${ }^{b}$ Jet Propulsion Laboratory, M/S 301-451, 4800 Oak Grove Drive, Pasadena, CA, 91109, USA \\ ${ }^{c}$ University of Michigan, Astronomy dept., 500 Church Street, Ann Arbor, MI 40109-1090, \\ USA \\ ${ }^{d}$ Institut d'Astrophysique Spatiale, Universit Paris-Sud, bâtiment 121, 91405, Orsay Cedex, \\ France \\ ${ }^{e}$ California Association for Research in Astronomy, 65-1120 Mamalahoa Hwy, Hilo, Hawaii, \\ 96743, USA

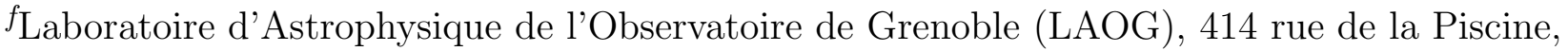 \\ BP 53-X, Grenoble, FRANCE \\ ${ }^{g}$ Michelson Science Center, California Institute of Technology, 770 S. Wilson Ave. MS 100-22, \\ Pasadena, CA 91125, USA \\ ${ }^{h}$ University of Massachusetts, Computer Science Department, Amherst, MA, 01003-4610 \\ ${ }^{i}$ Harvard-Smithsonian Center for Astrophysics, 60 Garden Street, Cambridge, MA, 02138, USA
}

\begin{abstract}
The infrared optical telescope array (IOTA), one of the most productive interferometers in term of science and new technologies was decommissioned in summer 2006. We discuss the testing of a low-resolution spectrograph coupled with the IOTA-3T integrated-optics beam combiner and some of the scientific results obtained from this instrument.
\end{abstract}

Keywords: infrared interferometry, infrared spectrograph, real-time software, stellar astronomy

\section{INTRODUCTION}

IOTA was a long-baseline optical interferometer located at the Smithsonian Institution's Whipple Observatory on Mount Hopkins, AZ, comprising three 45- $\mathrm{cm}$ diameter telescopes which could be positioned at 17 stations on an L-shaped track, where the arms were $15 \mathrm{~m}$ toward the south-east and $35 \mathrm{~m}$ toward the north-east. IOTA operated with 2 telescopes from 1995, and 3 telescopes from February 2002 to the end of June 2006. The interferometer was used as a testbed for new cutting-edge technologies, ${ }^{1,2}$ and produced astronomy results in the two-telescope and the three-telescope configurations. ${ }^{3-8,8-15}$ The three beams arriving from the vacuum delay-line tank hit three dichroic mirrors which separated the visible and infrared light. The visible beams continued toward the star tracker servo system. In the implementation discussed here, the infrared beams were reflected toward three flat mirrors and then three off-axis parabolas which focused the three beams on three single-mode (H-band) fibers feeding the IONIC-3T integrated-optics beam-combiner.

Interference was achieved inside the integrated optics component, resulting in three output pairs $\pi$ radians out of phase in intensity. The interference fringes were recorded while two of the dichroics were piezo-driven to scan a path of about $+50 \mu \mathrm{m}$ and $-50 \mu \mathrm{m}$, respectively, in order to scan through the fringe packet in the three

$\dagger$ Affiliated to Scottish Universities Physics Association, SUPA

Further author information: (Send correspondence to E.P.)

E.P: E-mail: ep41@st-and.ac.uk

Optical and Infrared Interferometry, edited by Markus Schöller, William C. Danchi, Françoise Delplancke Proc. of SPIE Vol. 7013, 70132V, (2008) · 0277-786X/08/\$18 · doi: 10.1117/12.789751 
beams. The six combined beams were then focused on six separate pixels of the PICNIC array ${ }^{2}$ and recorded as time series for science measurement. The same time series was used by the fringe-packet tracker. ${ }^{16}$ The path difference calculated by the packet-tracker was fed back to the piezo-scanning dichroics for a fast tracking response. The piezo scanners were off-loaded of their additional offsets every second, when a fraction of the error signal was sent to the short delay lines which were responsible for tracking the geometric delay caused by the rotation of the Earth.

The last upgrade to the instrument included the installation of a simple spectrograph using a non-deviating prism as dispersive element and a new readout mode for the infrared camera used for data acquisition. The spectrograph and infrared camera were moved to the Palomar testbed interferometer after the decommissioning of the IOTA interferometer in July 2006.

\section{THE IOTA SPECTROGRAPH}

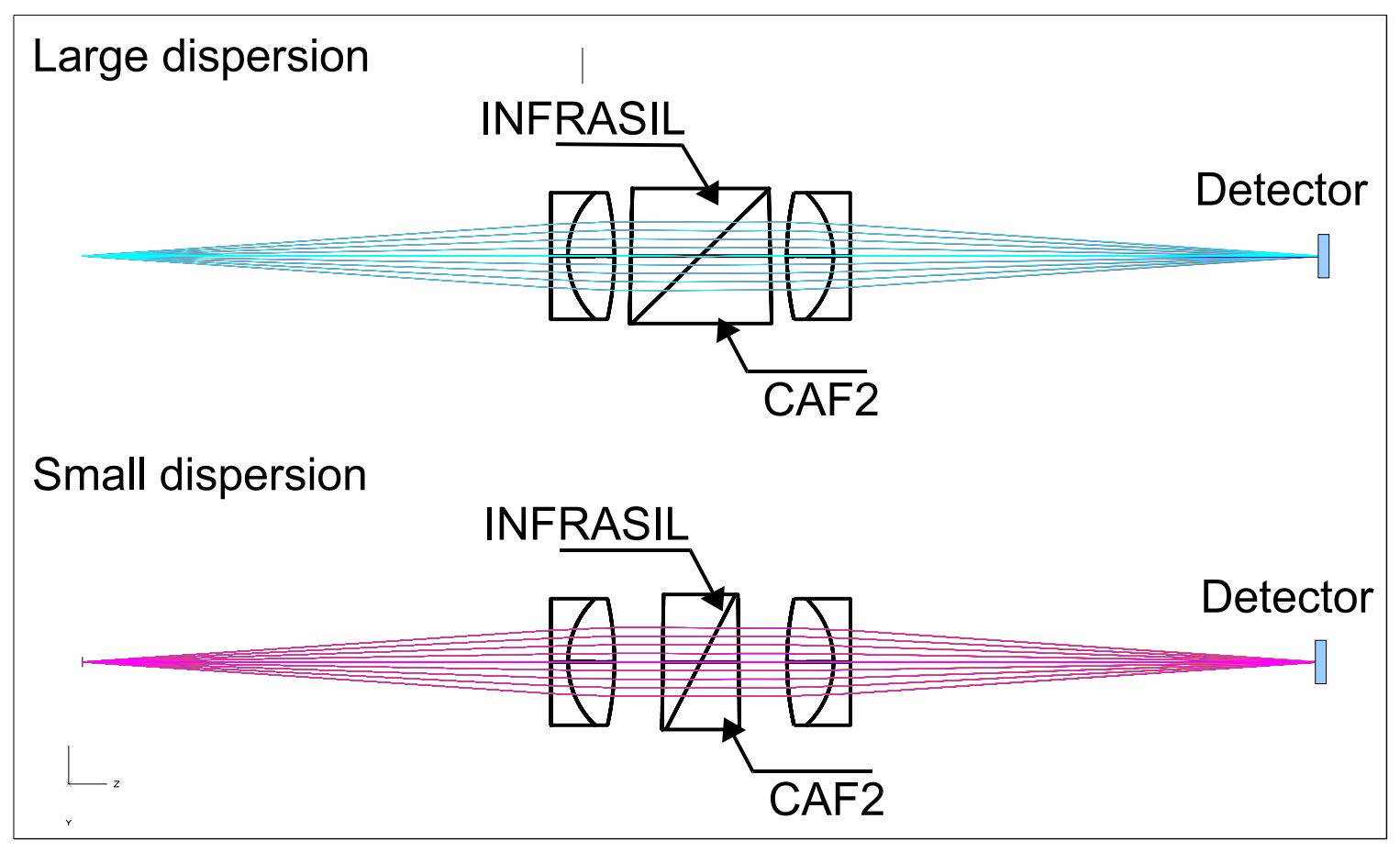

Figure 1. The most recent design of the IOTA spectrograph used two direct-view prisms as dispersive elements. The the six recombined pairs from the IONIC beam combiner are placed along the x-axis in this drawing, while the channels are dispersed in the $\mathrm{y}$-axis. The prisms could be easily interchanged in order to obtain either three or six chanelled spectra on the PICNIC infrared camera.

A design for a low-resolution infrared spectrograph for the IOTA interferometer was first discussed by Ragland. ${ }^{17}$ The idea behind the design was to build a simple prism-based spectrograph and use the existing optics and electronics of the IONIC beam combiner in order to build a cost-effective instrument. In this configuration it would be easy to switch from broad-band operation to chanelled-spectrum by simply interposing the dispersive elements in the pre-existing optical train of the beam combiner. While the concept was retained the final design did not include a deviating prism but a direct-view prism as shown in Figure 1

First fringes were acquired with the spectrograph on the star Alp Lyr the night the 19th of April 2006. Figure 4 shows seven dispersed IONIC channels in the vertical direction and three IONIC, subtracted in pairs, outputs on the horizontal axis. 




Figure 2. The spot diagram for the low-dispersion front-viewing prism when an $\mathrm{H}$-band filter is used. This configuration produced three spectral channels on the pixels of the PICNIC camera.

\subsection{Optical Design}

The optical ray-tracing of the IOTA spectrograph is shown in Figure 1. The four lenses represented in the drawing were doublets and were part of the original IONIC design described elsewhere. ${ }^{18}$ The prism were built in Infrasil-301 and $\mathrm{CaF}_{2}$ bonded with Norland 61 and anti-reflection coated on the two outer surfaces. The spectrograph was designed to work from J-band to K-band. Due to the sudden closure of the IOTA facility we only tested the $\mathrm{H}$-band operation mode by using the wide-band filter present in the filter wheel of the PICNIC infrared camera.

The six outputs of the IONIC beam combiner (left of Figure 1) were re-imaged on the pixels of the PICNIC infrared camera using the two original IONIC doublets, represented in Figure 1. The non-deviating prisms were simply mounted on a holder and placed in the middle of the optical train, between the doublets. The effect of placing a dispersive element in the optical train is simulated in the spot diagram of Figure 2 for the low-dispersion prism and Figure 3 for the high dispersion prism.

The low dispersion prism delivered three channels, across $\mathrm{H}$ band while the high dispersion prisms delivered six/seven channels across $\mathrm{H}$ band. The beam combiner and the optics of the spectrograph were at room temperature while the filter-wheel and the detector were in the original cryostat, cooled down at $\mathrm{LN}_{2}$ temperature. Minimum re-alignment and re-focusing of the optical channels on the detector were necessary when changing the configuration from low-to-high dispersion or from dispersed mode to wide-band mode. 


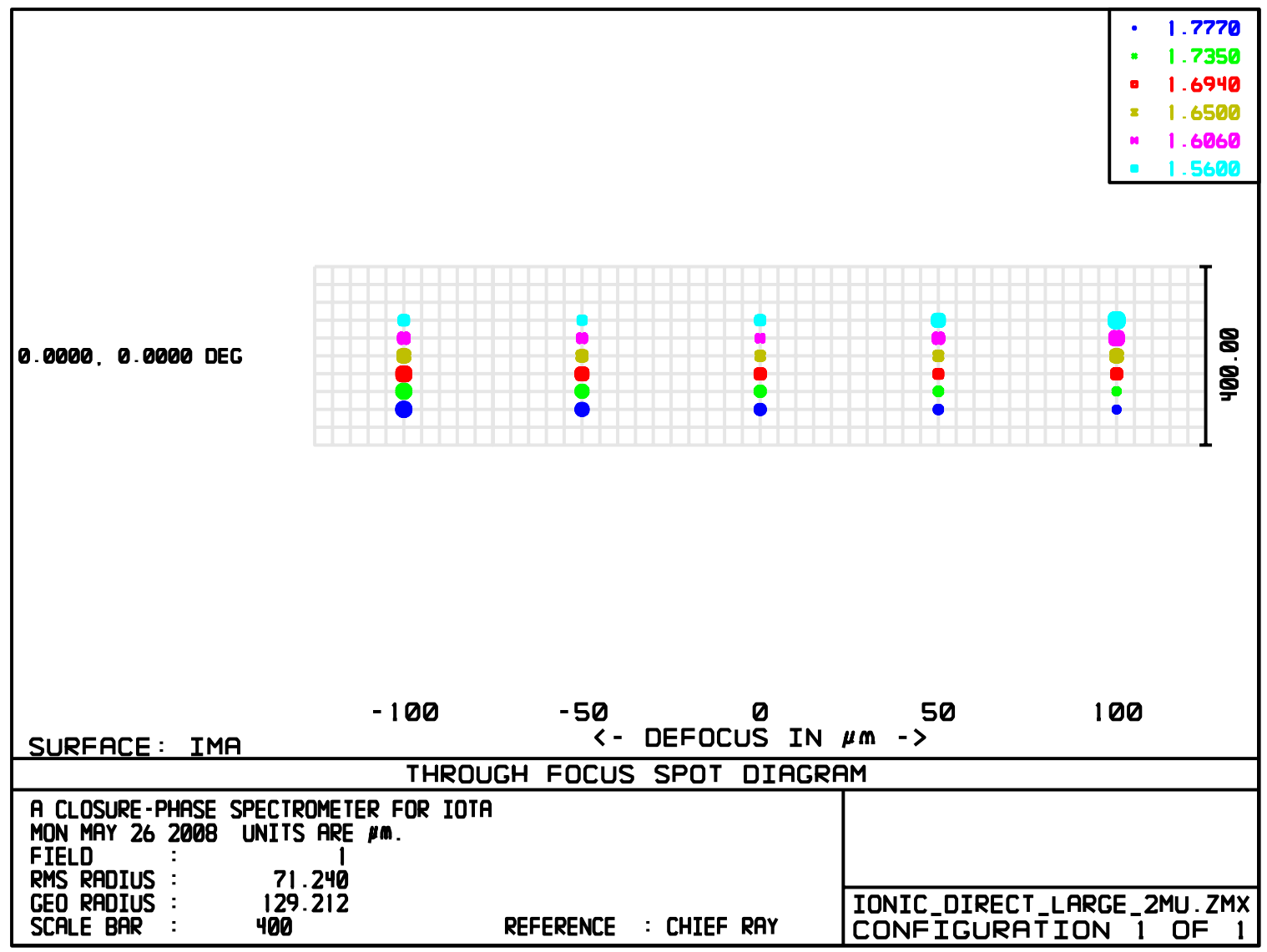

Figure 3. The spot diagram for the high-dispersion front-viewing prism when an $\mathrm{H}$-band filter is used. This configuration produced six spectral channels on the pixels of the PICNIC camera.

\subsection{Electronics and Software}

The original design ${ }^{2}$ of the complex programmable logic for device (CPLD) used for the control of the PICNIC camera was changed in order to allow the readout of $6 \times 6,6 \times 3$ and $6 \times 2$ channels instead of the original six non-dispersed IONIC outputs. The original digital circuit was limited in the number of pixels that could be read at the same time. For this reason the digital circuit was re-designed to be completely programmable and micro-instructions were developed to execute specialised micro-programmes capable of generating completely programmable readout sequences for the PICNIC infrared camera.

New software was developed to deal with the new capabilities of the CPLD controller and in order to generate the necessary microprogrammes encoding the read-out sequences "on-demand". The real-time code was also completely re-designed in order to accommodate the new readout modes: the high dispersion $6 \times 6$, the low dispersion $6 \times 3$, and a $6 \times 2$ mode capable acquiring twelve channels from a polarisation-splitting Wollaston prism. A backward-compatible six-pixel readout mode with no dispersion was also added for high-sensitivity, broad-band measurements needed for faint sources. The control interface of the instrument, running on a Unix workstation was re-designed to host the dispersed modes. The data pipeline developed for the IONIC beam combiner was also modified in order to host the new observing modes. Figure 5 shows reduced and calibrated visibilities from the modified pipeline for the star V Hya.

\section{RECENT SCIENCE}

We report here the observations of the cool-giant star V Hya and the symbiotic star CH Cyg. Part of these observation were also the very first data obtained from the IOTA spectrograph. The first published results from 

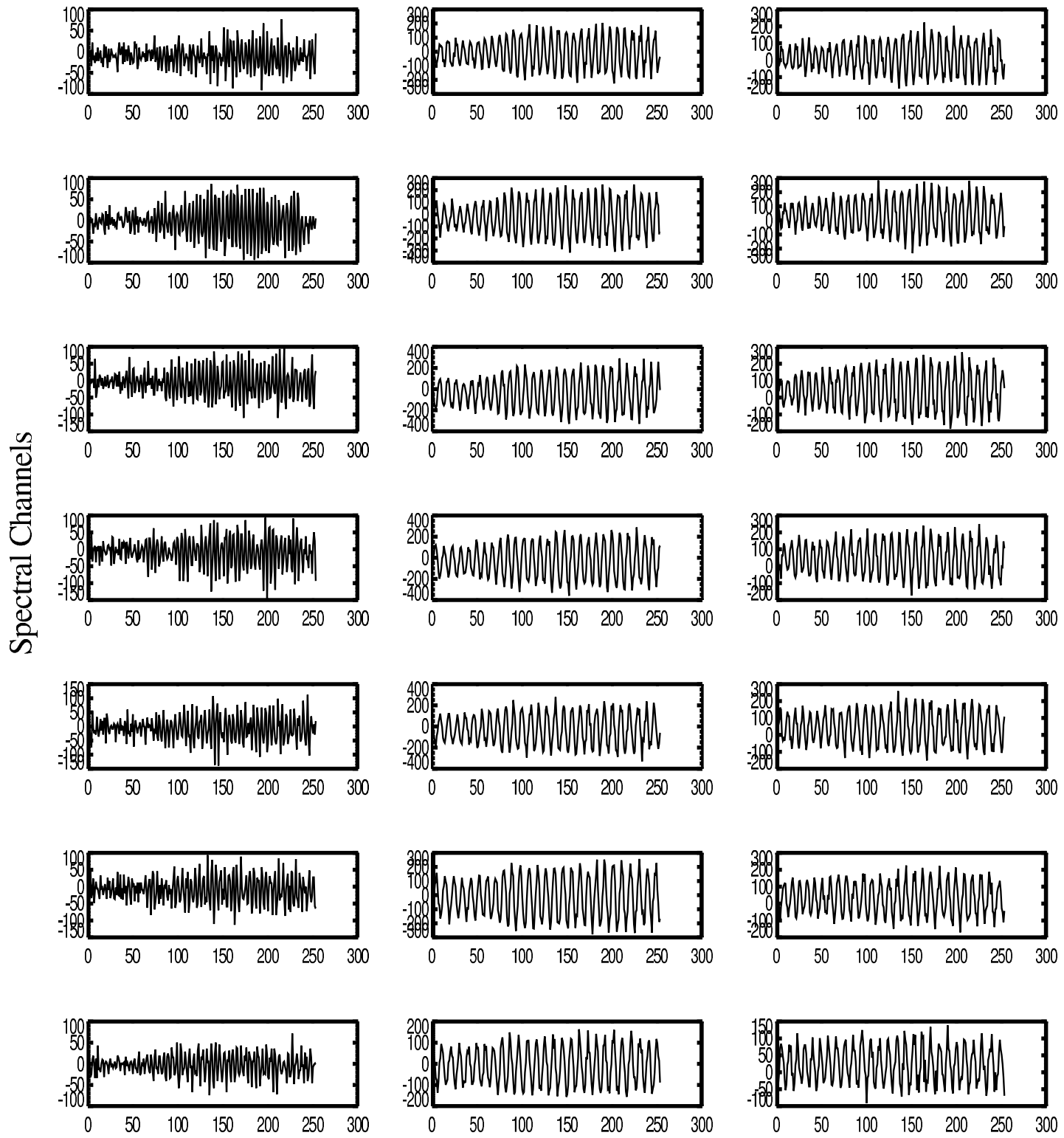

Figure 4. The first fringes obtained with the IOTA spectrograph on the star Alp Lyr in high-resolution configuration, the night of 19th April 2006. Seven spectral channels are shown in the vertical direction while fringes from each of the three pairs of the IONIC combiner are shown in the horizontal axis.

the spectrograph, reported on a refereed publications, are from Lacour et al. ${ }^{15}$

The observations were performed in 2004 using both the IOTA interferometer and an aperture masked Keck-1 telescope. Observations were performed on both stars using narrow-band filters at $\mathrm{H}$ band. We also obtained data in 2005 for CH Cyg. This data was acquired using a standard broadband H filter. Observations in 2006 were performed using the IOTA spectrograph for both stars.

Combining data from the IOTA interferometer and the Keck telescope with an aperture mask was successfully 



Figure 5. Visibility plots for six spectral channels obtained by the IOTA spectrograph for the star V Hya in 2006. Models representing the star and the dust are superposed to the data points. 
achieved in a study of dust shells around evolved stars. ${ }^{6}$ In this paper we apply the same technique in order to study the stellar and circumstellar environment of the stars V Hya and CH Cyg. We performed the observations as outlined in previous IOTA publications. ${ }^{19,20}$ We typically recorded series of 200 scans alternating between the science target and the calibrators. V Hya was observed together with several other targets which covered most of the observing nights.

Aperture masking converts the primary mirror of a telescope into an interferometric array, allowing the Fourier amplitudes and closure phases for a range of baselines to be recovered. ${ }^{21,22}$ For this work, we used a non-redundant aluminium "Golay" mask placed in front of the Keck-I infrared secondary mirror. ${ }^{23,24}$

For the observations performed at the Keck-1 telescope the Near InfraRed-Camera ${ }^{25}$ was used in a fast readout mode, adopting an integration time of $0.137 \mathrm{~s}$ per frame. The data recorded at $1.65 \mu \mathrm{m}$ were probably corrupted by highly-variable seeing and "wind shake", which blurs the fringes during the integration time and frustrates precise calibration. For this reason we azimuthally averaged the data-set from the Keck telescope. The asymmetries that we detected in the source were, in fact of the same magnitude as the error introduced by the wind shake and could be not reliably used to constrain large-scale asymmetries in the dust.

Several calibrators were employed as back-ups, to avoid unreported binary stars or sources subjected to time variability. We also cross-calibrated each calibrator with all the others, in order to check the consistency of the diameters with the literature and to verify night-to-night and across-the-night change in calibration.

\subsection{The Cool Giant Star V Hya}

$\mathrm{V}$ Hya is a high luminosity $\left(10^{4} \mathrm{~L} \odot\right)$ star classified as a normal N-type carbon star, its atmospheric $\mathrm{C} / \mathrm{O}$ abundance ratio being greater than unity. V Hya is placed on the infrared astronomical satellite (IRAS) colour-colour diagram as an asymptotic giant branch (AGB) star. Over a period of $10^{4}-10^{5}$ years these stellar objects eject about half of their mass in slowly expanding spherical winds. During a poorly understood and short-lived phase (100/1000 years) they transform from spherically symmetric AGB stars into aspherical planetary nebulae (PN).
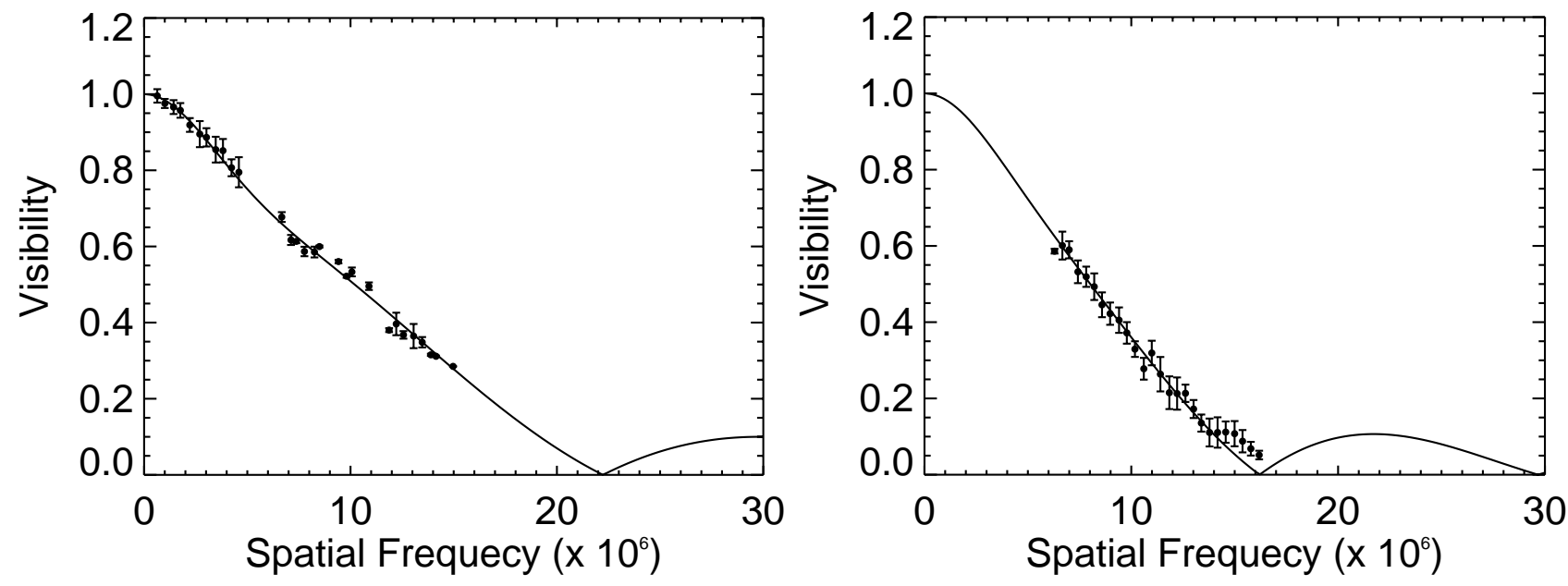

Figure 6. Visibility plots comprising all data from 2004 (left) and 2006 (right). The 2004 data on the left-hand side of the plots, with larger error bars is the aperture-masking data from Keck-1. Simple uniform disk plus a Gaussian disk models representing the star and the dust are superposed to the data points.

V Hya is a variable star: its light curve has a main period of 529 days consistent with Mira classification ${ }^{26}$ and an eclipse-like, secondary period of 6160 days. Knapp explains this secondary period as caused by extinction from a detached binary companion enshrouded by a thick cloud of obscuring dust. Sahai ${ }^{27}$ reports high speed 
jets $(\sim 100 \mathrm{~km} / \mathrm{s})$, which are normally inconsistent with low escape velocity of AGB stars but could be caused by the accretion of matter on the undetected companion.

Emission line broadening in optical spectra have also been detected indicating that the star is a fast rotator. ${ }^{28}$ This is inconsistent with slow rotation in AGB stars, an explanation being that the external envelope of the AGB star was spun up by the companion through tidal interaction. The line broadening could also be explained by turbulence in the circumstellar medium.

Here we present a set of narrow-band interferometric observations performed in 2004 in the H-band region of the infrared. The observations were performed with the IOTA-IONIC3 interferometer and the Keck telescope fitted with an aperture mask. Additional observations were obtained using the IOTA spectrograph in 2006. Although the uv coverage of the Keck aperture-mask was extensive and isotropic the coverage of the IOTA array was mostly concentrated in the North-West direction This did not allow us to use standard mapping software to obtain a direct image of the star.

Table 1. Size of the star and size of the dust shell for $\mathrm{V}$ Hya.

\begin{tabular}{ccccc}
\hline Year & Size Red-Giant (mas) & Size Dust & Flux ratio (RG/D) & $\chi 2$ \\
\hline 2004 & $11.3 \pm 0.1$ & $25.5 \pm 0.7$ & $3.1 \pm 0.2$ & 1.5 \\
2006 & $15.8 \pm 0.1$ & 25.5 & $4.2 \pm 0.3$ & 1.3 \\
\hline
\end{tabular}

We resorted then to create a simple model comprising of a uniform-disk component for the star and a Gaussian disk component for the dust. The result of our fit is illustrated Figure 6 . The most obvious result from the fit is that the size of the star increased from $11.3 \pm 0.1$ in 2004 to $15.8 \pm 0.1$ in 2006 . The size of the dust can be derived from the 2004 data-set, which included the low spatial frequencies from the aperture-masking experiment at the Keck telescope. The size of the dust was $25.5 \pm 0.7$ mas or $1.61 \pm 0.05$ stellar radii. The data from 2006 did not benefit from measurements from the Keck telescope, therefore the size of the dust in the fit is assumed to be the same as in 2004. The simple uniform plus Gaussian disk model seems to poorly fit our visibility data, indicating that the star may not be symmetric. This is also evident from the closure phase signature which is not reported here and will be part of a separate publication. The other result from this data-set is that the size of the star decreases with wavelength, as it can be shown in Figure 6.

\subsection{The Elusive Symbiotic Star CH Cyg}

Symbiotic stars are objects presenting combination spectra with the high excitation lines of a hot ionised nebula and the cool continuum absorption molecular features of a late-type star. Nowadays, symbiotic stars are understood as interacting binaries of short period, from a few to 10 years. The separation can vary from a few AU to slightly more than ten AU. The binary is usually composed of a cool giant star accreting a compact object, either a white dwarf or a neutron star.

$\mathrm{CH}$ Cyg is one of the most studied of symbiotic variables, due to the high luminosity and complexity of spectral and photometric behaviour. The star presents a composite spectra of a M6-7 giant star during quiescent phase and hot component blue continuum from 6000 to $9000 \mathrm{~K}$ temperature and low excitation line spectrum during the active phase. ${ }^{29}$ Webster $^{30}$ classified the star as an S-type symbiotic with no hot dust, but long term multi-wavelength photometry study of the star $^{31}$ have shown that hot dust appeared in the system after the 1984 outburst. The dust was modeled as spherical shell of inner radius of 15 AU by Bogdanov ${ }^{32}$ through the fitting of spectral energy distribution (SED).

The inclinations of the orbits are unknown. The inclinations usually assumed in the literature are based on the jet detected in radio ${ }^{33}$ which should be perpendicular to an accretion disk around the compact object. Crocker $^{34}$ warns that the jet cannot be used to determine the plane of the orbit because of its precessing nature. The precession of the jet is somewhat correlated with the two-year orbit implying perturbation of the orbit of the close pair. Also the assumption of the high inclination of the orbit ${ }^{35}$ may not hold if the eclipse is a grazing eclipse.

Due to limited uv plane coverage for all the epochs of our data, in particular for the 2005 epoch, we could not resort to direct imaging of the $\mathrm{CH}$ Cyg system. For this reason we used parametric modeling to derive the size 




Figure 7. Visibility plot comprising all data from 2004 to 2006 . The data on the left-hand side of the plot, with large error bars is the aperture-masking data from Keck-1. A simple uniform disk plus a Gaussian disk model representing the star and the dust is superposed to the data points. From this plot it is quite obvious that the size of the star did not change appreciably over three years.

of the star and the dust and the position and distance of the asymmetries detected in the closure-phase data. For model fitting we used publicly available least-squares minimisation routines*.

A simple model composed of a uniform disk (UD) for the star and a Gaussian disk (GD) for the dust was first attempted in order to obtain the size of the star and the dust. For this model all the data from all epochs was used since, by visual inspection, our visibility points superposed quite well, indicating that the size of the star did not change appreciably outside the error bars of the data, neither with time nor wavelength nor position angle. Figure 3 shows the result of the fit. The data was smoothed using an azimuthal average due to the otherwise very large number of data points present on the graph. For each bin we used the mean of the original data points weighted by their errors. The error on each new data point was the standard deviation for the bin. The fit was performed on the original and non-smoothed data. No attempt was done to fit the closure phase data at this point. Table 2 shows the parameters that were obtained from the fit. The value of $8.6 \pm 0.2$ mas, for the diameter of the red giant, agrees well within errors with the value obtained with infrared interferometry by Hofmann et al. ${ }^{36}$ The errors were derived using bootstrap statistics on the data set. A marginally improved $\chi^{2}$ was obtained by fitting an elliptical dust distribution around the star. However the difference in $\chi^{2}$ was too small in order to justify an asymmetric model for the dust in the near infrared. The parameters from the elliptical-dust model are also listed in Table 2 .

The size of the dust was found to be $17.1 \pm 0.2$ mas or $2.0 \pm 0.1$ stellar radii. The most interesting findings

${ }^{*}$ A non-linear least squares curve fitting (MPFIT), developed by Craig Markwardt.
http://cow.physics.wisc.edu/craigm/idl/ 
Table 2. Size of the star and of the dust shell.

\begin{tabular}{lccccr}
\hline Size Red-Giant (mas) & Size Dust & Flux ratio (RG/D) & P.A $\left(^{o}\right)$ & Axis ratio dust $(\mathrm{M} / \mathrm{m})$ & $\chi 2$ \\
\hline $8.6 \pm 0.2$ & $17.1 \pm 0.2$ & $10.0 \pm 0.3$ & 0.0 & 1.0 & 1.26 \\
$8.6 \pm 0.1$ & $17.1 \pm 0.1$ & $10.0 \pm 0.2$ & $0.15 \pm 0.3$ & $0.85 \pm 0.2$ & 1.24 \\
\hline
\end{tabular}

are about the asymmetries detected in the closure-phase signal in all the epochs of our data. This will not be discussed in this paper and will be the subject of a separate publication.

\section{CONCLUSIONS}

The IOTA interferometer was one of the most productive interferometers in term of science and new technologies in the history of optical and infrared interferometry. The sudden decommissioning of the instrument, the first of July 2006 virtually killed several on-going programmes of stellar astrophysics which could not be adapted to other interferometric facilities due to incompatible baselines, lack of sensitivity and the lack of access to the needed region of the sky. Efforts spent on the development of new instruments were wasted since very little data was acquired as a consequence of the IOTA shutdown.

We have reported here the development and first observations of a low-resolution spectrograph coupled with the IOTA-3T integrated-optics beam combiner. We have shown preliminary scientific results partly obtained with the help of the spectrograph on the cool-giant star V Hya and the symbiotic star CH Cyg.

The star V Hya increased its angular diameter from $11.3 \pm 0.1$ in 2004 to $15.8 \pm 0.1$ in 2006 . The size of the dust was $25.5 \pm 0.7$ mas or $1.61 \pm 0.05$ stellar radii. The size of the star was found to decreases with wavelength in the 2004 and 2006 data sets.

The star CH Cyg shows no change in angular diameter from 2004 to 2006. The value of $8.6 \pm 0.2$ mas, for the diameter of the red giant, agrees well within errors with the value obtained with infrared interferometry by Hofmann et al. ${ }^{36}$ The size of the dust was found to be $17.1 \pm 0.2$ mas or $2.0 \pm 0.1$ stellar radii.

\section{ACKNOWLEDGMENTS}

This research was made possible thanks to a Michelson Postdoctoral Fellowship, a Scottish Universities Physics Association (SUPA) advanced fellowship awarded to E. Pedretti and a Marie Curie Fellowship (within the Fifth Framework programme "Improving Human Research Potential and the Socio-economic Knowledge Base) granted to N. D. Thureau (contract HPMF-CT-200-00966). The IONIC project is a collaboration among the Laboratoire d'Astrophysique de Grenoble (LAOG), Laboratoire d'Electromagnetisme Microondes et Optoelectronique (LEMO), and also CEA-LETI and IMEP, Grenoble, France. The IONIC project is funded in France by the Centre National de Recherche Scientifique and Centre National d'Etudes Spatiales. This research has made use of NASA's Astrophysics Data System Bibliographic Services and of the SIMBAD. database operated at CDS, Strasbourg, France.

\section{REFERENCES}

[1] Monnier, J. D., Berger, J. P., Millan-Gabet, R., Traub, W. A., Carleton, N. P., Pedretti, E., Coldwell, C. M., and Papaliolios, C. D., "SMART Precision Interferometry at $794 \mathrm{~nm}$," in [Proc. SPIE, Interferometry for Optical Astronomy, Wesley A. Traub, editor], 4838, 1127-1138 (Aug. 2003).

[2] Pedretti, E., Millan-Gabet, R., Monnier, J. D., Traub, W. A., Carleton, N. P., Berger, J.-P., Lacasse, M. G., Schloerb, F. P., and Brewer, M. K., "The PICNIC Interferometry Camera at IOTA," PASP 116, 377-389 (Apr. 2004).

[3] Mennesson, B., Perrin, G., Chagnon, G., Foresto, V. C. d., Ridgway, S., Merand, A., Salome, P., Borde, P., Cotton, W., Morel, S., Kervella, P., Traub, W., and Lacasse, M., "Evidence for Very Extended Gaseous Layers around O-rich Mira Variables and M Giants," ApJ 579, 446-454 (Nov. 2002).

[4] Ohnaka, K., Beckmann, U., Berger, J.-P., Brewer, M. K., Hofmann, K.-H., Lacasse, M. G., Malanushenko, V., Millan-Gabet, R., Monnier, J. D., Pedretti, E., Schertl, D., Schloerb, F. P., Shenavrin, V. I., Traub, W. A., Weigelt, G., and Yudin, B. F., "JHK'-band IOTA interferometry of the circumstellar environment of R CrB," A\&A 408, 553-558 (Sept. 2003). 
[5] Monnier, J. D., Traub, W. A., Schloerb, F. P., Millan-Gabet, R., Berger, J.-P., Pedretti, E., Carleton, N. P., Kraus, S., Lacasse, M. G., Brewer, M., Ragland, S., Ahearn, A., Coldwell, C., Haguenauer, P., Kern, P., Labeye, P., Lagny, L., Malbet, F., Malin, D., Maymounkov, P., Morel, S., Papaliolios, C., Perraut, K., Pearlman, M., Porro, I. L., Schanen, I., Souccar, K., Torres, G., and Wallace, G., "First Results with the IOTA3 Imaging Interferometer: The Spectroscopic Binaries $\lambda$ Virginis and WR 140," ApJ 602, L57-L60 (Feb. 2004).

[6] Monnier, J. D., Millan-Gabet, R., Tuthill, P. G., Traub, W. A., Carleton, N. P., Coudé du Foresto, V., Danchi, W. C., Lacasse, M. G., Morel, S., Perrin, G., Porro, I. L., Schloerb, F. P., and Townes, C. H., "High-Resolution Imaging of Dust Shells by Using Keck Aperture Masking and the IOTA Interferometer," ApJ 605, 436-461 (Apr. 2004).

[7] Perrin, G., Ridgway, S. T., Coudé du Foresto, V., Mennesson, B., Traub, W. A., and Lacasse, M. G., "Interferometric observations of the supergiant stars $\alpha$ Orionis and $\alpha$ Herculis with FLUOR at IOTA," A\&A 418, 675-685 (May 2004).

[8] Kraus, S., Schloerb, F. P., Traub, W. A., Carleton, N. P., Lacasse, M., Pearlman, M., Monnier, J. D., Millan-Gabet, R., Berger, J.-P., Haguenauer, P., Perraut, K., Kern, P., Malbet, F., and Labeye, P., "Infrared Imaging of Capella with the IOTA Closure Phase Interferometer," AJ 130, 246-255 (July 2005).

[9] Perrin, G., Ridgway, S. T., Mennesson, B., Cotton, W. D., Woillez, J., Verhoelst, T., Schuller, P., Coudé du Foresto, V., Traub, W. A., Millan-Gabet, R., and Lacasse, M. G., "Unveiling Mira stars behind the molecules. Confirmation of the molecular layer model with narrow band near-infrared interferometry," A\&A 426, 279-296 (Oct. 2004).

[10] Millan-Gabet, R., Monnier, J. D., Berger, J.-P., Traub, W. A., Schloerb, F. P., Pedretti, E., Benisty, M., Carleton, N. P., Haguenauer, P., Kern, P., Labeye, P., Lacasse, M. G., Malbet, F., Perraut, K., Pearlman, M., and Thureau, N., "Bright Localized Near-Infrared Emission at 1-4 AU in the AB Aurigae Disk Revealed by IOTA Closure Phases," ApJ 645, L77-L80 (July 2006).

[11] Monnier, J. D., Berger, J.-P., Millan-Gabet, R., Traub, W. A., Schloerb, F. P., Pedretti, E., Benisty, M., Carleton, N. P., Haguenauer, P., Kern, P., Labeye, P., Lacasse, M. G., Malbet, F., Perraut, K., Pearlman, M., and Zhao, M., "Few Skewed Disks Found in First Closure-Phase Survey of Herbig Ae/Be Stars," ApJ 647, 444-463 (Aug. 2006).

[12] Zhao, M., Monnier, J. D., Torres, G., Boden, A. F., Claret, A., Millan-Gabet, R., Pedretti, E., Berger, J.-P., Traub, W. A., Schloerb, F. P., Carleton, N. P., Kern, P., Lacasse, M. G., Malbet, F., and Perraut, K., "Physical Orbit for $\lambda$ Virginis and a Test of Stellar Evolution Models," ApJ 659, 626-641 (Apr. 2007).

[13] Kraus, S., Balega, Y. Y., Berger, J.-P., Hofmann, K.-H., Millan-Gabet, R., Monnier, J. D., Ohnaka, K., Pedretti, E., Preibisch, T., Schertl, D., Schloerb, F. P., Traub, W. A., and Weigelt, G., "Visual/infrared interferometry of Orion Trapezium stars: preliminary dynamical orbit and aperture synthesis imaging of the $\theta^{1}$ Orionis C system," A\&A 466, 649-659 (May 2007).

[14] Ragland, S., Le Coroller, H., Pluzhnik, E., Cotton, W. D., Danchi, W. C., Monnier, J. D., Traub, W. A., Willson, L. A., Berger, J.-P., and Lacasse, M. G., "First Images of R Aquarii and Its Asymmetric $\mathrm{H}_{2} \mathrm{O}$ Shell," ApJ 679, 746-761 (May 2008).

[15] Lacour, S., Meimon, S., Thiebaut, E., Perrin, G., Verhoelst, T., Pedretti, E., Schuller, P. A., Mugnier, L., Monnier, J., Berger, J. P., Haubois, X., Poncelet, A., Le Besnerais, G., Eriksson, K., Millan-Gabet, R., Lacasse, M., and Traub, W., "The limb-darkened Arcturus; Imaging with the IOTA/IONIC interferometer," ArXiv e-prints 804 (Apr. 2008).

[16] Pedretti, E., Traub, W. A., Monnier, J. D., Millan-Gabet, R., Carleton, N. P., Schloerb, F. P., Brewer, M. K., Berger, J.-P., Lacasse, M. G., and Ragland, S., "Robust determination of optical path difference: fringe tracking at the Infrared Optical Telescope Array interferometer," Appl. Opt. 44, 5173-5179 (Sept. 2005).

[17] Ragland, S., Traub, W. A., Millan-Gabet, R., Carleton, N. P., and Pedretti, E., "A Low-resolution spectrograph for the IOTA interferometer," in [Interferometry for Optical Astronomy II. Edited by Wesley A. Traub. Proceedings of the SPIE, Volume 4838, pp. 1225-1233 (2003).], Traub, W. A., ed., Presented at the Society of Photo-Optical Instrumentation Engineers (SPIE) Conference 4838, 1225-1233 (Feb. 2003). 
[18] Berger, J.-P., Haguenauer, P., Kern, P. Y., Rousselet-Perraut, K., Malbet, F., Gluck, S., Lagny, L., SchanenDuport, I., Laurent, E., Delboulbe, A., Tatulli, E., Traub, W. A., Carleton, N., Millan-Gabet, R., Monnier, J. D., Pedretti, E., and Ragland, S., "An integrated-optics 3-way beam combiner for IOTA," in [Interferometry for Optical Astronomy II. Edited by Wesley A. Traub. Proceedings of the SPIE, Volume 4838, pp. 1099-1106 (2003).], Traub, W. A., ed., Presented at the Society of Photo-Optical Instrumentation Engineers (SPIE) Conference 4838, 1099-1106 (Feb. 2003).

[19] Coude Du Foresto, V., Ridgway, S., and Mariotti, J.-M., "Deriving object visibilities from interferograms obtained with a fiber stellar interferometer," A\&AS 121, 379-392 (Feb. 1997).

[20] Millan-Gabet, R., Schloerb, F. P., and Traub, W. A., "Spatially Resolved Circumstellar Structure of Herbig AE/BE Stars in the Near-Infrared," ApJ 546, 358-381 (Jan. 2001).

[21] Baldwin, J. E., Haniff, C. A., Mackay, C. D., and Warner, P. J., "Closure phase in high-resolution optical imaging," Nature 320, 595-597 (Apr. 1986).

[22] Jennison, R. C., "A phase sensitive interferometer technique for the measurement of the Fourier transforms of spatial brightness distributions of small angular extent," MNRAS 118, 276-+ (1958).

[23] Monnier, J. D., "Infrared interferometry and spectroscopy of circumstellar envelopes," Ph.D. Thesis (1999).

[24] Tuthill, P. G., Monnier, J. D., Danchi, W. C., Wishnow, E. H., and Haniff, C. A., "Michelson Interferometry with the Keck I Telescope," PASP 112, 555-565 (Apr. 2000).

[25] Matthews, K., Ghez, A. M., Weinberger, A. J., and Neugebauer, G., "The First Diffraction-Limited Images from the W. M. Keck Telescope," PASP 108, 615-+ (July 1996).

[26] Knapp, G. R., Dobrovolsky, S. I., Ivezić , Z., Young, K., Crosas, M., Mattei, J. A., and Rupen, M. P., "The light curve and evolutionary status of the carbon star V Hya," A\&A 351, 97-102 (Nov. 1999).

[27] Sahai, R., Morris, M., Knapp, G. R., Young, K., and Barnbaum, C., "A collimated, high-speed outflow from the dying star V Hydrae," Nature 426, 261-264 (Nov. 2003).

[28] Barnbaum, C., Morris, M., and Kahane, C., "Evidence for Rapid Rotation of the Carbon Star V Hydrae," ApJ 450, 862-+ (Sept. 1995).

[29] Deutsch, A. J., Lowen, L., Morris, S. C., and Wallerstein, G., "The Spectrum of CH Cygni from 1961 to 1973," PASP 86, 233-+ (June 1974).

[30] Webster, B. L. and Allen, D. A., "Symbiotic stars and dust," MNRAS 171, 171-180 (Apr. 1975).

[31] Taranova, O. G. and Iudin, B. F., "Photometric observations of CH Cygni in the period 1978-1987 - Dust matter condensation on its circumstellar envelope during 1985-1987," Ap\&SS 146, 33-39 (July 1988).

[32] Bogdanov, M. B. and Taranova, O. G., "Parameters of the Dust Envelope of the Symbiotic Star CH Cygni," Astronomy Reports 45, 797-803 (Oct. 2001).

[33] Taylor, A. R., Seaquist, E. R., and Mattei, J. A., "A radio outburst and jet from the symbiotic star CH CYG," Nature 319, 38-41 (Jan. 1986).

[34] Crocker, M. M., Davis, R. J., Spencer, R. E., Eyres, S. P. S., Bode, M. F., and Skopal, A., "The symbiotic star CH Cygni - III. A precessing radio jet," MNRAS 335, 1100-1108 (Oct. 2002).

[35] Skopal, A., Bode, M. F., Lloyd, H. M., and Tamura, S., "Eclipses in the symbiotic system CH CYG," A\&A 308, L9-L12 (Apr. 1996).

[36] Hofmann, K.-H., Beckmann, U., Berger, J.-P., Bloecker, T., Brewer, M. T., Lacasse, M. G., Malanushenko, V., Millan-Gabet, R., Monnier, J. D., Ohnaka, K., Pedretti, E., Schertl, D., Schloerb, F. P., Scholz, M., Traub, W. A., Weigelt, G., and Yudin, B., "Near-infrared IOTA interferometry of the symbiotic star CH Cyg," in [Interferometry for Optical Astronomy II. Edited by Wesley A. Traub . Proceedings of the SPIE, Volume 4838, pp. 1043-1046 (2003).], Traub, W. A., ed., Presented at the Society of Photo-Optical Instrumentation Engineers (SPIE) Conference 4838, 1043-1046 (Feb. 2003). 